\title{
On the Performance of Multiple Description Codes over Bit Error Channels
}

\author{
Helge Coward ${ }^{12}$ \\ Dept. of Telecommunications \\ Norwegian University of Sci. and Tech. \\ 7491 Trondheim, Norway \\ e-mail: cowardQtele.ntnu.no
}

\author{
Raymond Knopp ${ }^{1}$ \\ Dept. of Mobile Communications \\ Institut EURECOM \\ 06904 Sophia-Antipolis, France \\ e-mail: Raymond.KnoppQeurecom. $f x$
}

\author{
Sergio D. Servetto \\ Audiovisual Communications Lab. \\ Swiss Federal Institute of Technology \\ 1015 Lausanne, Switzerland \\ e-mail: servetto@lcavsun1.epfl.ch
}

\begin{abstract}
Multiple description (MD) codes have been proposed to provide robustness against data loss in packet-switched networks. In this paper, we report results on the performance of $M D$ codes when used on bit error channels with error correcting codes, in order to handle loss of blocks when the number of errors in a block exceeds the correcting ability of the code. We find that MD codes appear to be outperformed by a more traditional system in which source and channel code parameters are jointly optimized to minimize end-to-end distortion.
\end{abstract}

\section{INTRODUCTION}

Multiple description (MD) source coding [1], [2] is a method for sending different information over two channels so that it is decodable from any one channel, but higher quality decoding is possible using both channels. The MD coding problem was originally formulated as a problem of communication over packet networks. However, loss of data can occur in any communications system that uses error correcting codes when the amount of errors exceeds the error correcting abilities of the code. Shannon's channel coding theorem states that for sufficient rate, there exist codes with arbitrarily small probability of decoding error, but there are at least two examples of situations where this is not applicable. One example is when delay constraints prevent the use of long codes. Another example is communication over channels for which a lower bound on the error probability exists for codes of any length, e.g., the block fading case [3]. The performance of MD coding on such channels is investigated.

Our main goal is to assess if and when MD codes make sense to be used in this context. For that purpose, we study a memoryless Gaussian source and mean square error distortion measure. We consider two bit-error channels: a pair of independent binary symmetric channels (BSC), and a pair of independent block fading channels (BFC). To make our evaluation, we consider two systems. One system uses an MD code that produces two descriptions, each of which is channel coded independently and transmitted on one of the channels. The other system uses a traditional single description (SD) coder, whose output is channel encoded and spread over the two channels. For the BSC, a block code with adjustable protection performing according to the Gilbert bound [4], a uniform scalar quantizer with an optimal entropy coder, and in the MD case, a practical index assignment [2] is assumed in the performance calculations. For the BFC, the channel code performance is modeled as the lower bound on achievable block error probabilities with (SD) and without (MD) interleaving [5], and the source code performance is modeled as the rate-distortion performance [1]. The MD source coder

\footnotetext{
${ }^{1}$ The work was carried out at EPFL

${ }^{2}$ Supported by the Research Council of Norway
}

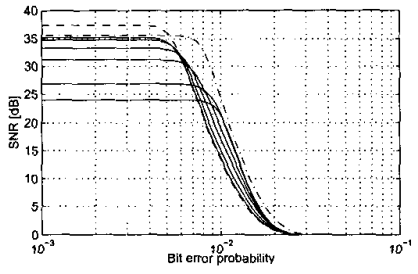

Solid MD coder with varying side and central distortion and $(1000,810,31)$ channel code Dashed SD coding with $(1000,810,31)$ channel code Dash-dot SD coding with $(1000,771,39)$ code and thus lower source bit rate

Fig. 1: Performance of MD and SD systems with a fixed channel code at a total rate of 8 bits/sample.

can handle the loss of one description, but if both descriptions are lost, or if any block is lost in the SD coding system, the decoder outputs the source mean.

\section{Performance Comparisons}

Comparisons have been made between SD and MD coding, where the channel quality and the total rate (number of channel symbols per source symbol) is constant. The results show that in almost all situations, SD coding is superior to MD coding. For the BFC, interleaving is more efficient than MD coding. For the BSC, the result can be explained from Fig. 1, which shows that improving the MD coder performance when decoding from one channel at the expense of the quality when decoding from both channels reduces the performance for good channels and improves it for poor channels. SD coding with the same error correcting code is better than all the MD codes for good channels and worse for poor channels. However, if the protection in the channel coder is increased at the expense of source coder fidelity, SD coding can outperform all the MD coder systems for all channel qualities.

\section{Concluding Remarks}

In this work, we have reported only one representative result of a study in which we analyzed the usefulness of MD codes to deal with loss of data in communication systems with noise as the major channel impairment. More experiments and some interpretation of these results can be found in the longer version submitted for review of this paper, available from http://lcavwwr.epfl.ch/"servetto/publications/isit2001b.html

\section{REFERENCES}

[1] L. H. Ozarow, "On a source coding problem with two channels and three receivers," Bell Syst. Tech. J., vol. 59, pp. 1909-1921, Dec. 1980.

[2] V. A. Vaishampayan, "Design of multiple description scalar quantizers," IEEE Trans. Inform. Theory, vol. 39, pp. 821-834, May 1993.

[3] R. Finopp and P. A. Humblet, "On coding for block fading channels," IEE'E Trans. Inform. Theory, vol. 46, pp. 189-205, Jan. 2000.

[4] R. E. Blahut, Theory and Practice of Data Transmission Codes. Dept. ECE, Univ. of Illinois, Urbana-Champaign, 2nd ed. (preliminary draft), 1997.

[5] L. H. Ozarow, S. Shamai, and A. D. Wyner, "Information theoretic considerations for cellular mobile radio," IEEE Trans. Veh. Technol., vol. 43, pp. 359-378, May 1994. 